\title{
Multi-scale Characterization of White Matter Tract Geometry
}

\author{
Peter Savadjiev ${ }^{1,2}$, Yogesh Rathi², Sylvain Bouix ${ }^{2}$, \\ Ragini Verma ${ }^{3}$, and Carl-Fredrik Westin ${ }^{1}$ \\ ${ }^{1}$ Laboratory for Mathematics in Imaging and ${ }^{2}$ Psychiatry Neuroimaging Laboratory \\ Brigham and Women's Hospital, Harvard Medical School, Boston, MA, USA \\ ${ }^{3}$ Section of Biomedical Image Analysis, Dept. of Radiology, University of \\ Pennsylvania, Philadelphia, PA, USA
}

\begin{abstract}
The geometry of white matter tracts is of increased interest for a variety of neuroscientific investigations, as it is a feature reflective of normal neurodevelopment and disease factors that may affect it. In this paper, we introduce a novel method for computing multi-scale fibre tract shape and geometry based on the differential geometry of curve sets. By measuring the variation of a curve's tangent vector at a given point in all directions orthogonal to the curve, we obtain a $2 \mathrm{D}$ "dispersion distribution function" at that point. That is, we compute a function on the unit circle which describes fibre dispersion, or fanning, along each direction on the circle. Our formulation is then easily incorporated into a continuous scale-space framework. We illustrate our method on different fibre tracts and apply it to a population study on hemispheric lateralization in healthy controls. We conclude with directions for future work.
\end{abstract}

\section{Introduction}

The brain consists of diverse structures, each with a characteristic shape and an intricate architecture. Their shape varies across the normal population, and is an important feature thought to reflect genetic and environmental factors that may contribute to disorders of neurodevelopmental origin or neurodegenerative diseases (e.g., [1]). In this context, the study of white matter geometry is of importance to the neuroscience of white matter and disorders that affect it.

A large group of methods for white matter geometry analysis in diffusion MRI compute the curvature and torsion of individual fibres recovered with a tractography algorithm (e.g., 2]). The geometry of sets of curves is usually obtained by mapping individual curves to medial axes/surfaces (e.g. [3]) or an average representation (e.g., 45]). However, this type of mapping may involve heuristic decisions in the choice of corresponding points and fibre similarity measures. An elegant alternative was recently introduced on the basis of the currents framework [6], which represents fibre tracts as a smooth vector field and captures global tract shape while avoiding the need for specific point correspondences [7.

All geometry analysis methods based on fibre tractography are inherently limited in that tractography does not, in general, produce stable and reproducible 
results. Recognizing this limitation, a method for computing white matter geometry indices directly from diffusion imaging data without requiring prior tractography was proposed in [8]. This method, however, is currently only defined for the single tensor model of diffusion, itself with well known limitations.

In the present work, we propose a scale-based white matter geometry analysis method that is situated logically between that of [8], and vector field based methods such as 7]. While our method is based on vector fields derived from tractography and is therefore subject to all associated limitations, its advantage with respect to [8] is that it is not limited by the tensor model of diffusion, and also allows for a more precise characterisation of fibre fanning at different spatial scales, as detailed in Section 1.1. As for the currents method of [7, it is optimized to capture global tract shape and its modes of variation in a population. In contrast, our method computes local geometrical features based on the differential geometry of curve sets, which makes possible subsequent tractbased statistical analysis with methods such as [3]. We note, however, that our method is complementary to the currents framework of [7], and both may be used in conjunction in order to analyse the geometry of a currents vector field.

\subsection{Comparison to Tensor-Based Model of Dispersion}

Both our method and the method of [8] compute macrostructural white matter geometry. However, they approach this problem in diametrically opposed ways, which makes them complementary to each other. The method of [8] works directly with DTI data within a 3D neighborhood, without any prior knowledge about fibre tracts. This is an advantage, as it allows to avoid tractography and its limitations. This, however, comes at a price: the geometry of all the white matter present in the 3D neighborhood is represented by a single scalar, which may be less informative when distinct fibre populations pass near each other.

In contrast, our new method works with the tangent vector field of tracts obtained by tractography. This reliance on tractography is a limitation, but it also allows the analysis of a specific tract independently of the influence of other nearby tracts. Furthermore, no constraints are imposed on the underlying model of diffusion. Unlike the method of [8], defined only for diffusion tensor fields, our method can be used even with high quality HARDI data. Finally, instead of computing a single scalar to represent fibre dispersion at a point, we compute directional dispersion in a "dispersion distribution function", as detailed below.

The two methods therefore exploit the two sides of a basic trade-off: avoiding the uncertainty inherent in tractography [8, vs. exploiting information about tract structure provided by tractography, as proposed here.

\section{Geometrical Framework}

The work in 9] models white matter fibre geometry by associating an orthonormal frame $\left(E_{T}, E_{N}, E_{B}\right)$ with each point along each $3 \mathrm{D}$ curve. Here $E_{T}$ is the curve's tangent vector, and $E_{N}$ and $E_{B}$ are the normal and binormal vectors. The 
local tract geometry can then be characterized, with respect to the local frame, by computing the variation of $E_{T}$ in the frame's three orthonormal directions.

In this work, we propose to measure the rate of change of the tangent vector $E_{T}$ not only in the direction of the normal $E_{N}$ and the binormal $E_{B}$, but in all directions in the plane orthogonal to $E_{T}$. This allows to avoid the need to define Frenet frames, which can be unstable. By computing the rate of change of $E_{T}$ along the entire circle orthogonal to $E_{T}$, instead of only 2 directions, we insure against missing "interesting events", and we describe more completely the complex geometry of white matter tracts. The function on a circle thus computed is a 2D "dispersion distribution function" (DDF), i.e. a function on the circle with values proportional to the amount of fibre dispersion in each direction orthogonal to the fibre. This function provides a richer description of dispersion than the scalar measure of [8], and constitutes our main contribution.

\section{Approach}

\subsection{Problem Statement}

The goal of our method is the following: given a set $\mathcal{C}$ of curves $C_{i}$ that represent the output of some streamline tractography algorithm, with a tangent vector $E_{T}$ defined at each point $\mathbf{p}$ on each curve $C_{i} \in \mathcal{C}$, we compute the function

$$
\Psi(\theta)=\nabla_{\mathbf{v}} E_{T} .
$$

Here $\nabla_{\mathbf{v}} E_{T}$ represents the covariant derivative of the tangent vector $E_{T}$ in direction $\mathbf{v}$, with the constraints that $\left\{\mathbf{v} \mid \mathbf{v} \in \mathcal{S}^{1} \bigcap \mathbf{v} \perp E_{T}\right\}$, i.e., $\mathbf{v}$ is a direction on the unit circle centered at $\mathbf{p}$ in the plane orthogonal to $E_{T}$, and is denoted by angle $\theta$ in this plane. This function is computed at each $\mathbf{p}$ along each $C_{i}$, resulting in a DDF at each $\mathbf{p}$ describing the local fibre dispersion pattern relative to the local tangent vector $E_{T}$, as illustrated in Fig. 1 (left).

We note that (11) is defined only for continuous vector fields. That is, a line passing though point $\mathbf{p}$ on curve $C_{i}$ with direction $\mathbf{v}$ must always intersect another curve $C_{j}$ at point $\mathbf{p}^{\prime}$ with a tangent vector $E_{T}^{\prime}$ defined at $\mathbf{p}^{\prime}$, otherwise $\nabla_{\mathbf{v}} E_{T}$ will not exist. Since we work with a vector field $F=\bigcup_{\mathcal{C}} E_{T}$ of tangent vectors of $1 \mathrm{D}$ curves in $3 \mathrm{D}$ Euclidean space $\mathbb{R}^{3}, F$ is not continuous, specific point correspondences cannot always be established and (1) is not always defined.

To resolve this issue, we construct a continuous vector field $F^{c}(x, y, z)$ at each $(x, y, z) \in \mathbb{R}^{3}$ by averaging $F$ over a neighborhood $\mathcal{N}$ centered at $(x, y, z)$ :

$$
F^{c}(x, y, z)=\frac{1}{N} \sum_{(i, j, k) \in \mathcal{N}} F_{(i, j, k)}
$$

where $\mathcal{N}$ is some neighborhood of $\mathbb{R}^{3}$ centered at location $(x, y, z)$, and $N$ is the number of vectors of $F$ that occur within $\mathcal{N}$.

We note that $F$ is discrete because it is obtained from a discrete set of curves, but also because of the discrete nature of diffusion MRI data, which causes 


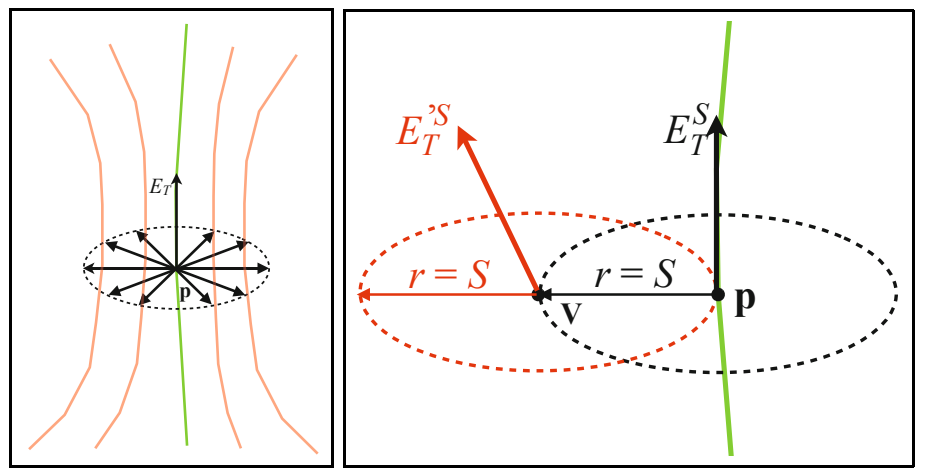

Fig. 1. LefT: Our method computes at each point $\mathbf{p}$ a function on the unit circle orthogonal to the tangent vector $E_{T}$ at $\mathbf{p}$ which measures the rate of change of $E_{T}$ in all of these directions, thus computing a 2D "dispersion distribution function". RIGHT: To compute $\nabla_{\mathbf{v}} E_{T}$ at location $\mathbf{p}$ at scale $S$, we define a disk-shaped neighborhood with radius $S$ around $\mathbf{p}$, over which vector field $F=\bigcup_{\mathcal{C}} E_{T}$ is averaged. The same averaging is performed over a neighborhood centered at $\mathbf{p}+S \mathbf{v}$ (orange). $\nabla_{\mathbf{v}} E_{T}$ is then approximated as the angular difference between the two vectors thus obtained.

each curve $C_{i}$ not to be continuous, but rather a polyline approximation to a continuous curve. Each $C_{i}$ is therefore a sequence of small linear segments, with one tangent vector $E_{T}$ per segment. There is thus a countable number of vectors belonging to $F$ in each neighborhood $\mathcal{N}$, which allows the summation in (2). The spatial location of these vectors is denoted with the $(i, j, k)$ subscript in (2), which are floating point coordinates with sub-voxel resolution.

We also note that a priori, the tangent vectors can be presented either as $E_{T}$ or as $-E_{T}$. To avoid arbitrary sign changes from one location to the next which can drastically affect the result of (2), we ensure a sign consistency over the entire dataset relative to a global coordinate frame, such as that derived from the principal components of the tracts' spatial distribution.

\subsection{Scale Space}

Since we seek to characterize the variation of curve orientation in directions orthogonal to the curves, in our implementation we choose the neighborhood $\mathcal{N}$ to be shaped as a disk lying in the plane orthogonal to $E_{T}$, with a small thickness chosen to be 1 voxel. The radius of this disk is treated as a scale parameter. To compute (1) for a given direction $\mathbf{v}$ at a given location $\mathbf{p}$, we first apply (2) at $\mathbf{p}$ in order to obtain a value for $E_{T}$ at scale $S, E_{T}^{S}$. Then, we apply (2) at location $\mathbf{p}+S \mathbf{v}$ (i.e., at a distance $S$ from $\mathbf{p}$ in direction $\mathbf{v}$ ), in order to obtain a value for the tangent vector $E_{T}^{\prime} S$ at location $\mathbf{p}+S \mathbf{v}$. Finally, we approximate $\nabla_{\mathbf{v}} E_{T}$ (at scale $S$ ) as the angular difference between $E_{T}^{S}$ and $E_{T}^{\prime S}$ :

$$
\nabla_{\mathbf{v}} E_{T} \approx \arccos \left(\left\langle E_{T}^{S}, E_{T}^{\prime S}\right\rangle\right)
$$

with $\langle.,$.$\rangle denoting the standard dot product in \mathbb{R}^{3}$. 


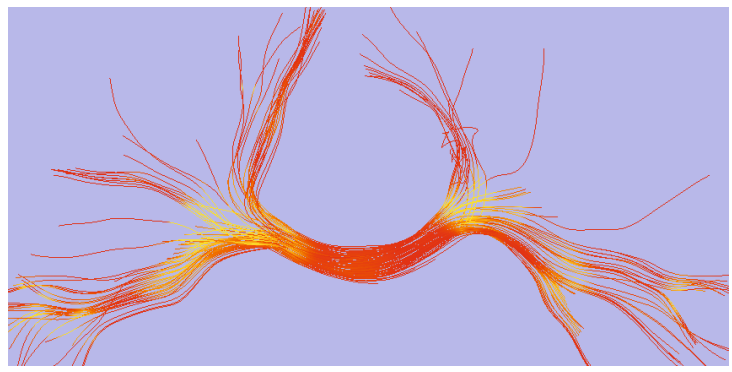

Fig. 2. The total dispersion (TD) visualized for tracts forming part of the corpus callosum. Here and in subsequent figures, yellow indicates high TD values.

These steps are illustrated in Fig. 1 (right). The inclusion of a scale parameter in this manner allows for a simple way to construct a continuous scale space.

\section{Validation}

Our method is a high-level analysis that is applied after tractography. We assume the particular tractography method has already been tested on synthetic data or phantoms, and its limitations are known. The focus of the experiments in this paper is the macrostructural geometric analysis of already-traced fibre tracts.

Our method was tested on tracts traced with a filtered tractography algorithm for HARDI data [10. This method was run on diffusion-weighted imaging data acquired from a volunteer on a GE Signa HDxt 3.0T scanner using an echo planar imaging sequence with a double echo option, an 8 Channel coil and ASSET with a SENSE-factor of 2. The acquisition consisted of 51 directions with $\mathrm{b}=900$ $\mathrm{s} / \mathrm{mm}^{2}$, and 8 images with $\mathrm{b}=0 \mathrm{~s} / \mathrm{mm}^{2}$, with scan parameters $\mathrm{TR}=17000 \mathrm{~ms}$, $\mathrm{TE}=78 \mathrm{~ms}, \mathrm{FOV}=24 \mathrm{~cm}, 144 \times 144$ encoding steps, $1.7 \mathrm{~mm}$ slice thickness. 85 axial slices covering the whole brain were acquired.

As described previously, our method computes 2D DDFs at each point along each curve in the tract set. In order to summarize this large amount of information and present it visually, in the following figures we show fibres where each point is colored by a measure of total dispersion (TD), such that yellow indicates high TD values. We define TD as the average value of $\Psi(\theta)$ (11) at a point.

In Fig. 2, we show the TD measure for a set of fibres passing through the corpus callosum. Here TD was computed with a scale parameter $S=5 \mathrm{~mm}$. As expected, the measure is highest in regions with highest overall dispersion.

We next explore the effect of varying the scale parameter $S$ on a fibre tract which connects the substantia nigra of the brain stem to the caudate nucleus, a sub-cortical grey matter structure. This tract was selected for illustration purposes because it presents a well-defined fanning structure.

In Fig. 3, we present views of this tract colored by TD, computed at scales $S$ ranging from $1.7 \mathrm{~mm}$ to $13.6 \mathrm{~mm}$. At the smallest scale only very local dispersion features are highlighted. With increasing scale, the larger fanning structure is highlighted more strongly, while small features are progressively lost. 


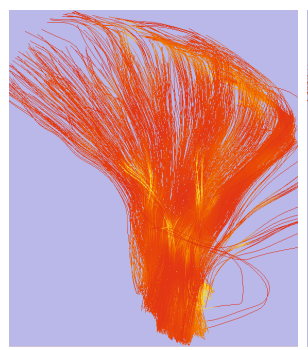

(a) $S=1.7 \mathrm{~mm}$

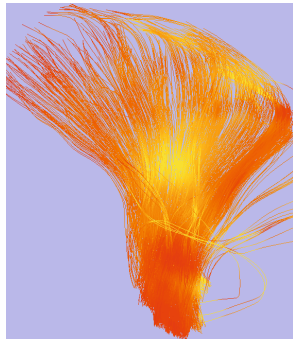

(e) $S=8.5 \mathrm{~mm}$

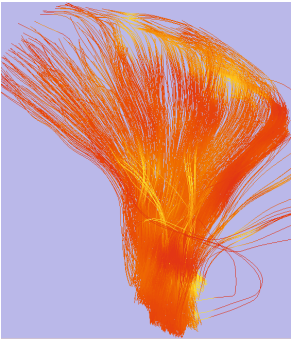

(b) $S=3.4 \mathrm{~mm}$

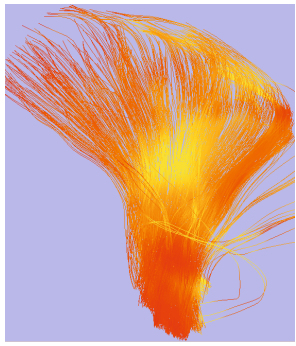

(f) $S=10.2 \mathrm{~mm}$

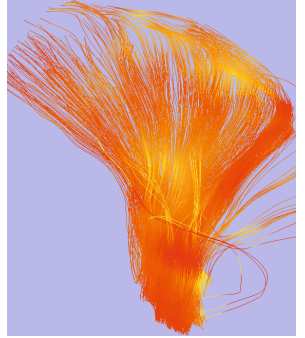

(c) $S=5.1 \mathrm{~mm}$

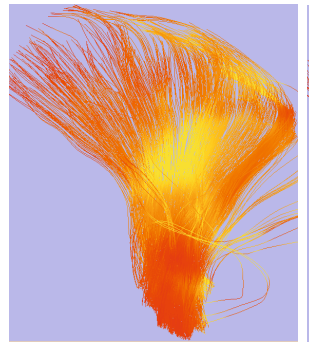

(g) $S=11.9 \mathrm{~mm}$

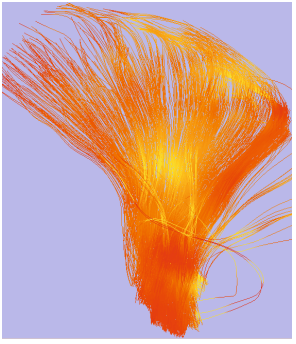

(d) $S=6.8 \mathrm{~mm}$

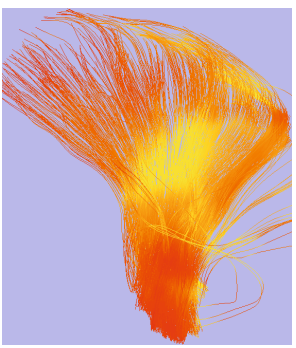

(h) $S=13.6 \mathrm{~mm}$

Fig. 3. A fibre tract colored by TD, for a range of values for scale parameter $S$

\section{A Study of Lateralization in Healthy Controls}

We illustrate the applicability of the method to population studies via an investigation of lateralization in healthy controls. Diffusion MRI data was acquired from 16 adult healthy male volunteers using the protocol described in Section 4 .

Our study was focused on tracts associated to the inferior frontal cortex, as this area has been shown to be lateralized in healthy males, for example in terms of functional connectivity [11, and it has also been implicated in disorders such as autism and schizophrenia (e.g., [1]). We extracted fibre tracts connecting the pars orbitalis cortical area (part of the inferior frontal cortex) of each hemisphere, using the filtered HARDI tractography algorithm of [10]. We first computed whole-brain tractography, from which we extracted the interhemispheric tracts connecting both cortical regions as defined by an automated FreeSurfer (http://surfer.nmr.mgh.harvard.edu) parcellation of the cortex. The tracts were then cut within $5 \mathrm{~mm}$ from the midsagittal plane. For each subject, we computed the mean TD at scale $S=5 \mathrm{~mm}$ over these tracts in each hemisphere. An example of these tracts for one subject is shown in Fig. 4 .

The lateralization results are presented in Fig. 5 and they indicate an overall increase of TD in the right hemisphere, with a p-value of 0.030 (two-tailed T test). The male inferior frontal cortex has been previously shown to be rightlateralized in terms of volume and functional connectivity (e.g., [11]). Anatomical differences in white matter geometry are less well known, and our method can be 

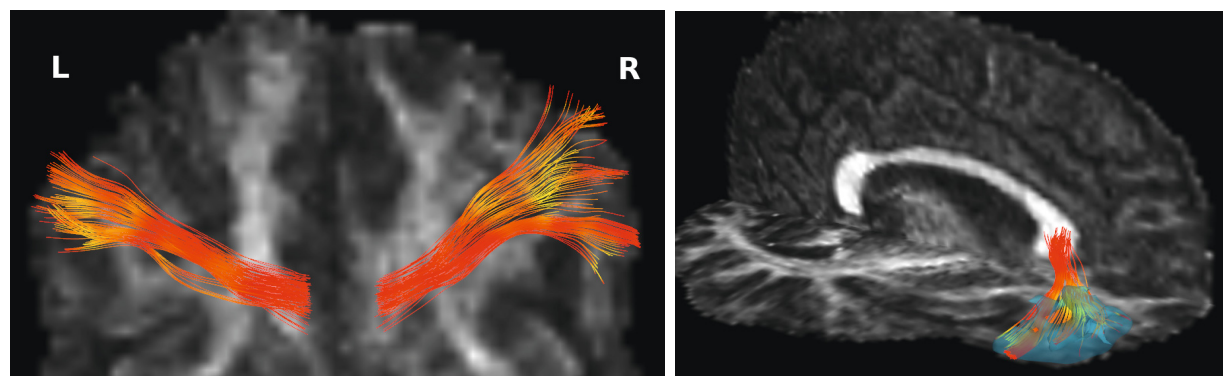

Fig. 4. LEFT: The set of tracts used in our group study shown for one subject. The fibres are colored by TD (computed at scale $S=5 \mathrm{~mm}$ ), and are overlaid on an axial slice through the FA volume. RIGHT: The same fibre tract shown with both a sagittal and an axial slice though FA, as well as a semi-transparent model (blue) of the FreeSurfer cortical segmentation of the pars orbitalis.

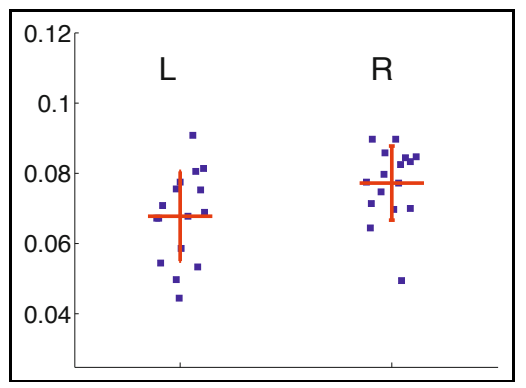

Fig. 5. Comparison of the mean TD value (units of $\mathrm{rad} / \mathrm{mm}$ ) for each individual, shown for each hemisphere. A t-test between the two groups yields a $p$-value of $p=0.030$. Horizontal red lines: group mean. The error bars indicate \pm 1 standard deviation. $\mathrm{L}$ : left hemisphere. R: right hemisphere.

used in this context. While our current results are preliminary, they do illustrate the applicability of the method to population studies.

\section{Discussion and Conclusion}

In this paper, we presented a multi-scale approach for computing white matter fibre geometry, based on the local differential geometry of curve sets. The method works with curves traced by fibre tractography algorithms, and has both advantages and weaknesses. The main drawback of this method is its dependence on the quality of the tractography algorithm used to generate the fibres. With this limitation in mind, we reviewed in Section 1.1 several advantages of our method relative to a non-tractography method such as [8]. In addition, we note that fibre tracts provide an explicit correspondence between the white matter and locations on the cortical surface where they originate or terminate. This is very 
important for establishing a connection between white matter and grey matter geometry, as the relationship between the two may provide novel insights into a variety of neuroscientific applications, regarding for example brain development or atrophies caused by disease. We will address these questions in future work.

Finally, we note that the TD measure reduces the information in $\Psi(\theta)$ to a directionless scalar. In order to better take advantage of the directional information contained in $\Psi(\theta)$, one may define an inner product between $\Psi(\theta)$ and a vector field, in order to measure dispersion in a specific direction. We will analyse such directional information in future work.

Acknowledgements. Work supported by NIH grants R01MH092862, R01MH074794, R01MH082918, P41RR013218, and P41EB015902.

\section{References}

1. Toga, A.W., Thompson, P.M.: Mapping brain asymmetry. Nature Rev. Neurosci. 4, 37-48 (2003)

2. Batchelor, P.G., Calamante, F., Tournier, J.D., Atkinson, D., Hill, D.L.G., Connelly, A.: Quantification of the shape of fiber tracts. Magn. Res. in Medicine 55, 894-903 (2006)

3. Yushkevich, P.A., Zhang, H., Simon, T.J., Gee, J.C.: Structure-specific statistical mapping of white matter tracts. NeuroImage 41, 448-461 (2008)

4. Corouge, I., Fletcher, P.T., Joshi, S., Gouttard, S., Gerig, G.: Fiber tract-oriented statistics for quantitative diffusion tensor MRI analysis. Medical Image Analysis $10(5), 786-798(2006)$

5. O'Donnell, L., Westin, C.F.: Automatic tractography segmentation using a highdimensional white matter atlas. IEEE Trans. Medical Imaging 26(11), 1562-1575 (2007)

6. Vaillant, M., Glaunès, J.: Surface Matching via Currents. In: Christensen, G.E., Sonka, M. (eds.) IPMI 2005. LNCS, vol. 3565, pp. 381-392. Springer, Heidelberg (2005)

7. Durrleman, S., Pennec, X., Trouvé, A., Ayache, N.: Statistical models on sets of curves and surfaces based on currents. Medical Image Analysis 13(5), 793-808 (2009)

8. Savadjiev, P., Kindlmann, G.L., Bouix, S., Shenton, M.E., Westin, C.F.: Local white matter geometry from diffusion tensor gradients. NeuroImage 49, 3175-3186 (2010)

9. Savadjiev, P., Zucker, S.W., Siddiqi, K.: On the differential geometry of 3D flow patterns: Generalized helicoids and diffusion MRI analysis. In: Proc. IEEE Intl. Conf. on Computer Vision, ICCV 2007 (2007)

10. Malcolm, J.G., Shenton, M.E., Rathi, Y.: Filtered multi-tensor tractography. IEEE Trans. on Medical Imaging 29, 1664-1675 (2010)

11. Tomasi, D., Volkow, N.D.: Laterality patterns of brain functional connectivity: Gender effects. Cerebral Cortex 22(6), 1455-1462 (2012) 